\title{
Questions Today: Sistema m-learning como auxílio ao ensino da matemática
}

\author{
Denise Maciel Sena ${ }^{1}$, Elaine Harada T. de Oliveira ${ }^{1}$, Leandro S. G. de Carvalho ${ }^{1}$ \\ ${ }^{1}$ Instituto de Computação - Universidade Federal do Amazonas (UFAM) \\ CEP 2.223 - 69.077-000 - Manaus - AM - Brasil \\ dms@icomp.ufam.edu.br, elainedicomp.ufam.edu.br, \\ galvaodicomp. ufam.edu.br
}

\begin{abstract}
This article presents an experience report on the evaluation of the application of Questions Today - a mobile learning app to support math learning - developed from the analysis of m-learning applications used in teaching mathematics in the literature. The application covers specific math content and is applied to students through a fixation exercise. The focus is on high school level and the deployment was conducted in a public school located in Manaus-AM, using the mobile phone.
\end{abstract}

Resumo. Este artigo apresenta um relato de experiência sobre a avaliação da aplicação do Questions Today - um aplicativo de aprendizagem móvel de apoio à aprendizagem de matemática - desenvolvido a partir da análise de aplicativos m-learning utilizados no ensino de matemática, encontrados na literatura. A aplicação aborda conteúdos específicos da matemática e avalia os alunos através de um exercício de fixação. O foco está no nível de ensino médio e a implantação foi realizada em uma escola pública estadual localizado na cidade de Manaus-AM, usando o celular como dispositivo móvel.

\section{Introdução}

Em junho de 1999, ministros da educação de 29 países que então compunham a Comunidade Europeia firmaram a Declaração de Bolonha. Esse documento estabeleceu dois conceitos que nortearam as ações da Comunidade Europeia, no sentido de facilitar a integração nos aspectos educacionais. $\mathrm{O}$ primeiro conceito foi o de estabelecer critérios e parâmetros para que os sistemas educacionais dos países da Comunidade permitissem aos cidadãos o reconhecimento de seus diplomas em todo território europeu. $\mathrm{O}$ segundo conceito permitia o deslocamento de estudantes para aprendizagem e treinamento, e também de professores e burocratas. Foram esses dois parâmetros que deram origem ao conceito de mobile learning (m-learning) - literalmente - aprendizagem móvel, ou aprendizagem em movimento. Ao mesmo tempo em que se desenvolveu todo o esforço conjunto para integração, a partir de 1999, o conceito de m-learning, na Comunidade Europeia deixou de abranger apenas a ideia de aprendizagem para incorporar também a de mobilidade. Cada vez mais o m-learning passou a designar a aprendizagem com a utilização de telefones celulares, pequenos computadores pessoais (PDAs) e, eventualmente, laptops em redes sem fio. [Figueiredo et al. 2011]

As práticas educativas vêm sendo aprimoradas a cada dia, para que se possa acompanhar o desenvolvimento do aluno envolvido no processo de ensino $\mathrm{e}$ aprendizagem. Docentes da disciplina de matemática, tinham a convicção durante anos 
que as dificuldades causadas na aprendizagem no ensino superior, herdaram-se exclusivamente pelos problemas existentes no ensino básico. Na procura por soluções à aprendizagem da matemática, podem existir contribuições através dos dispositivos móveis [Batista et al. 2010]. Em um mundo cada vez mais interconectado, serviços digitais são, cada vez mais, frequentes. Surgem, a todo momento, novas demandas educacionais. Através de diversas ferramentas, um maior número de pessoas tem acesso à aprendizagem móvel (m-learning) [Figueiredo et al. 2011].

É importante prezar pela aprendizagem de forma dinâmica e motivadora. Dessa forma, os recursos desenvolvidos devem conter multimídia e interatividade. É preciso, então, que o estudante consiga, com o auxílio do software ou aplicativo, trabalhar a construção de novos conteúdos, desenvolvendo um ciclo de aprendizagem [Marçal et al. 2010].

Neste contexto este artigo apresenta o relato de experiência da avaliação da aplicação do Questions Today - um sistema m-learning (mobile learning) de apoio a aprendizagem de matemática - desenvolvido a partir da análise de aplicativos m-learning utilizados no ensino de matemática com conteúdos e recursos diferenciados, utilizados nos diferentes níveis de ensino (fundamental, médio e superior), encontrados na literatura.

O artigo está organizado em seis seções, incluindo esta introdução. A Seção 2 apresenta brevemente conceitos referentes a m-learning e matemática e alguns trabalhos relacionados. A Seção 3 apresenta o sistema desenvolvido. A Seção 4 apresenta a avaliação realizada e os resultados obtidos. A Seção 5 apresenta as considerações finais, e é seguida pelas referências bibliográficas.

\section{Aprendizagem Matemática: M-Learning e Matemática}

Neto \& Fonseca (2013) definem mobile learning (m-learning) como um conceito novo que envolve a mobilidade e o estudante, o qual pode realizar atividades educacionais sem a limitação do espaço físico de um ambiente de ensino. Pode-se aprender em ambientes fora das salas de aula, basta apenas motivação de aprender e um dispositivo móvel. Como benefícios da m-learning podem ser apontados:

- Melhorar os recursos para o aprendizado do aluno, o qual os utilizará importantes para execução de tarefas, como por exemplo, consulta de informações via Internet, utilização da câmera digital, gravação de sons, troca de mensagens e outras funcionalidades existentes;

- Acessar conteúdos didáticos em qualquer lugar e a qualquer momento, de acordo com a conectividade do dispositivo;

- Aumentar a interatividade com professores e as estratégias de aprendizado disponíveis, através de novas tecnologias que dão suporte tanto à aprendizagem formal quanto à informal; e

- Fornecer meios para o desenvolvimento de métodos inovadores de ensino e de treinamento, utilizando os novos recursos de computação e de mobilidade.

A tecnologia funciona como um complemento na educação. Explorando este conceito, os dispositivos móveis tornam a educação portável de um ambiente para outro, como da sala de aula para a casa do estudante. Assim, permite que o potencial da aprendizagem seja contínuo. No entanto, a aprendizagem móvel não se destina a substituir 
o aprendizado em sala de aula, mas pode ser visto, como um acessório que aumenta as possibilidades e estímulos para a aprendizagem. E defendem que aprendizagem móvel não aponta para um tipo diferente de aprendizagem, mas uma aprendizagem comum em condições e ambientes diferentes.

Barcelos \& Batista (2010) defendem a importância do uso consciente de tecnologias como recurso pedagógico no processo de ensino e aprendizagem de matemática e a necessidade de formação apropriada para este fim. Relata que as instituições de ensino têm papel fundamental na formação de cidadãos aptos a usufruir os benefícios das Tecnologias de Informação e Comunicação (TIC). Em particular, na Matemática, as TIC podem favorecer a compreensão de conceitos, o desempenho na resolução de problemas e o desenvolvimento do raciocínio lógico-dedutivo do aluno.

Tendo em vista o uso de dispositivos móveis na aprendizagem de Matemática, na literatura podem ser encontrados alguns trabalhos relacionados a este aqui apresentado. Marçal (2010) aponta utilizar dispositivos móveis é uma interessante ferramenta de auxílio ao ensino e à aprendizagem, e relata a experiência da criação de três aplicações m-learning a partir um conjunto de requisitos necessários ao desenvolvimento, e estabelecimento de um processo guia que pode servir como uma referência prática para a criação de softwares m-learning em matemática. Além disso, o artigo apresenta as aplicações e proposta para realizar testes com alunos de ensino médio, para avaliar a implementação de tais requisitos, impactos do uso e a viabilização do aprendizado matemático.

Barcelos \& Batista (2010), relatam um estudo piloto utilizando um aplicativo específico para celular, Graph2Go, em uma experiência presencial. O referido estudo ocorreu em novembro de 2009, com 4 horas de duração, tendo como objetivo promover a análise de transformações gráficas de funções, utilizando o Graph2Go. O público alvo foi composto por alunos do Ensino Superior do IF Fluminense: oito alunos de Engenharia de Controle e Automação Industrial e quatro alunos da Licenciatura em Matemática. Um dos pontos relevantes deste trabalho é que além do teste piloto, ele apresenta projetos com o objetivo de incentivar o uso pedagógico das TIC, tendo em vista a melhoria do processo de ensino e aprendizagem de Matemática no Ensino Médio.

Neto \& Fonseca (2013) propõe a utilização de jogos educativos para dispositivos móveis como meio de estimular o aprendizado da Matemática. Para tanto, foi criado um jogo baseado na obra literária de Malba Tahan: $\mathrm{O}$ homem que calculava. Os participantes do experimento realizado com o jogo foram dezesseis alunos oriundos de duas escolas do Recife, sendo quatro alunos de uma escola particular (cursando o $8^{\circ}$ ano do ensino fundamental) e doze alunos de uma escola pública estadual (cursando o $1^{\circ}$ ano do ensino médio). Para conseguir respostas aos questionamentos, ao final do jogo, foi aplicado um questionário individualmente para cada aluno, o que resultou em muitos pontos positivos. Além disso, o artigo aponta que abordagens que fogem do comum acabam despertando maiores interesses nos alunos.

Diante das características apresentadas nos trabalhos relacionados, esta pesquisa tem como diferencial a execução da junção dessas diferentes características, do desenvolvimento de um sistema para auxiliar o ensino e à aprendizagem, utilizar o sistema desenvolvido em uma experiência presencial como fator motivacional para atrair os alunos e aumentar o seu interesse para o conteúdo apresentado e avaliar a aceitabilidade da aplicação como uma abordagem diferenciada com intuito de despertar 
interesse no aluno no seu processo de ensino-aprendizagem da matemática.

\section{Sistema de apoio à aprendizagem móvel}

Os dispositivos móveis são considerados uma nova dimensão na educação, principalmente por permitirem o aprendizado em contextos específicos, facilmente extensíveis ao mundo real [Marçal et al. 2010]. Para as necessidades de inovações no processo de ensino e aprendizagem no contexto matemático, uma das possíveis soluções é o uso ou o desenvolvimento de aplicativos m-learning relacionados a aprendizagem matemática.

Assim, como parte da análise do processo de desenvolvimento, foi realizado um mapeamento sistemático - é um método atualmente bastante utilizado para se ter uma visão geral, ampla e organizada da área a ser pesquisada [Petersen et al., 2008; Kitchenham, 2004] - onde, inicialmente as publicações foram selecionadas de acordo com critérios de inclusão e exclusão estabelecidos durante o protocolo do mapeamento.

Em uma análise inicial, 129 publicações foram analisadas a partir do primeiro filtro, onde foram examinados o título, resumo e palavras-chave. Se fossem considerados relevantes para o tema de pesquisa, a publicação era pré-selecionada. Em uma segunda análise, retornadas do primeiro filtro, apenas 8 publicações foram examinadas por completo, extraindo-se os dados das aplicações m-learning com referências à aprendizagem de matemática, que auxiliou o levantamento de requisitos como: a pequena existência de sistemas com mais de um recurso implementado; o interesse de apresentar multiconteúdos ao invés de conteúdos específico; nível de ensino mais utilizado para a aplicação de avaliações foi o nível médio e as tecnologias utilizadas relacionadas a plataformas e/ou sistemas operacionais, destacando-se mais a mobile e uma pequena porcentagem utilizando sistemas operacionais Android. Com a análise dos requisitos mais relevantes extraídos das aplicações, foi desenvolvido um sistema educativo para dispositivos móveis com objetivo de apresentar conceitos básicos de matemática de uma forma diferente, com situações e problemas que o aluno precisasse mobilizar conhecimentos desenvolvidos em sala de aula.

O referido aplicativo foi desenvolvido durante a elaboração de um trabalho de conclusão de curso, onde o processo de desenvolvimento teve os seguintes objetivos: Análise, Implementação e Testes de Implantação. Em razão de ser um sistema m-learning, o mesmo foi desenvolvido por um conjunto composto por linguagens (HTML5 ${ }^{1}$, $\mathrm{CSS3}^{2} /$ Responsive Web Design ${ }^{3}$ e Java Script ${ }^{4}$ ) utilizadas no desenvolvimento de sistemas e aplicando as técnicas mais avançadas de CSS3 utilizadas no desenvolvimento de sistemas WEB/MOBILE, e por este motivo, além de ter acesso em interface desktop sua aparência se adapta a qualquer dispositivo móvel, portanto, independente de plataforma, para realizar a instalação do sistema era apenas necessário um navegador web instalado no dispositivo móvel.

O sistema foi desenvolvido com uma interface disposta a facilitar o acesso aos conteúdos disponíveis no sistema. Na tela principal como mostra a Figura 1 a seguir, são apresentados dois botões que podem dar acesso ao Menu do sistema e o botão de Avançar

\footnotetext{
${ }^{1}$ http://www.w3.org/html/

${ }^{2}$ http:// coyote.icomp.ufam.edu.br/tizen/css3.pdf

${ }^{3} \mathrm{http}: / /$ sergiolopes.org/responsive-web-design/

${ }^{4}$ http:// coyote.icomp.ufam.edu.br/tizen/javascript.pdf
} 
que apresenta os conteúdos disponíveis na aplicação.

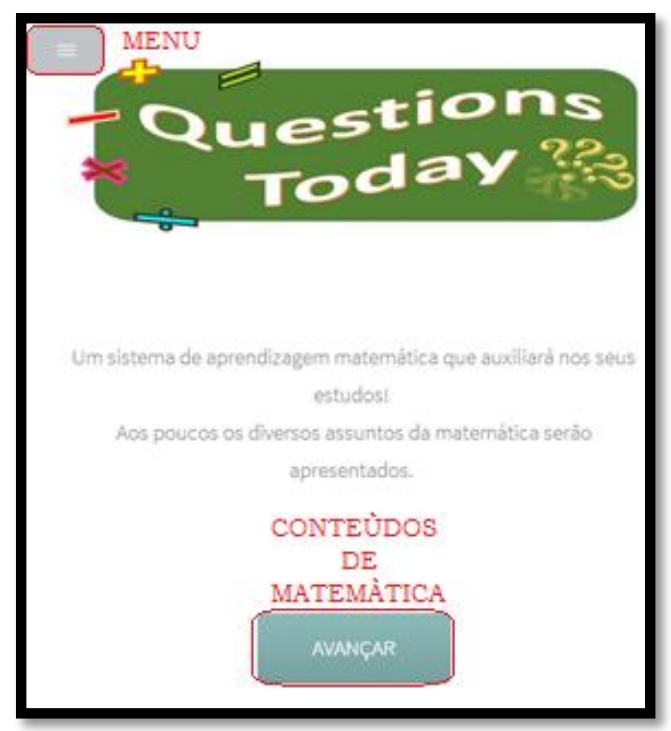

Figura 2. Tela inicial do sistema Questions Today

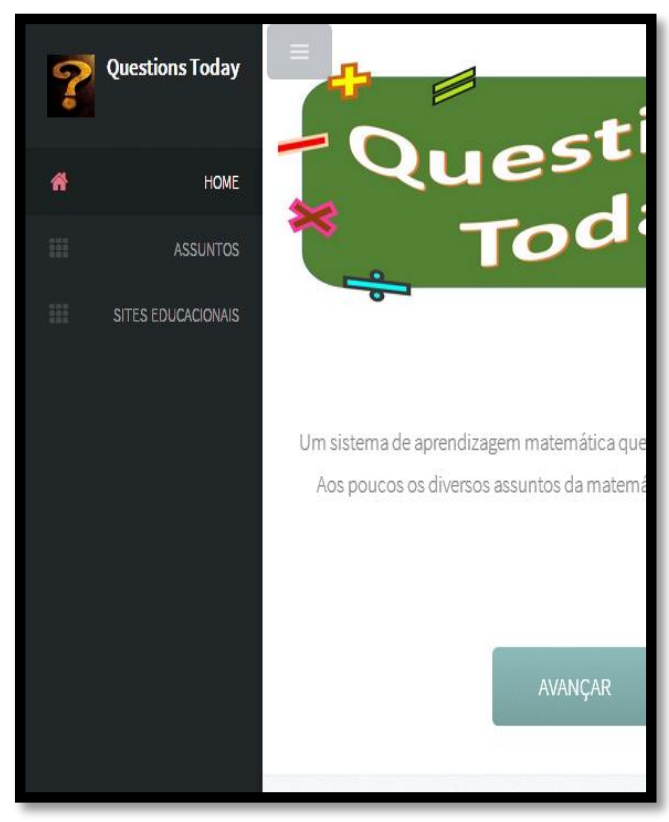

Figura 1. Tela de seleção do Menu

Com os conteúdos apresentados no sistema, o aluno poderá escolher uma opção a ser explorada como mostra a Figura 4 a seguir, e o sistema apresentará o conteúdos com imagens explicativas e ao término um Exercício de Fixação.
Quando o menu for selecionado, é possível visualizar as opções disponíveis no sistema, como mostra a Figura 2 a seguir.

Após o menu ser selecionado, fica a critério do aluno escolher a opção apresentada no sistema (Home, Assuntos, Sites Educacionais). Caso a opção escolhida seja HOME, o sistema será redirecionado a Tela Inicial do sistema.

Os objetivos principais deste sistema envolvem: promover aos alunos do ensino médio um melhor aproveitamento dos conteúdos abordados em sala de aula, introduzir conhecimentos básicos da matemática e estimular o processo de ensino e aprendizagem do aluno. Portanto, caso o aluno selecione a opção Avançar presente na tela inicial, é possível visualizar os assuntos relativos a disciplina de matemática, apresentado na Figura 3 a seguir. Da mesma forma, caso a opção de ASSUNTOS seja escolhida no menu, o sistema redirecionará a esta tela.

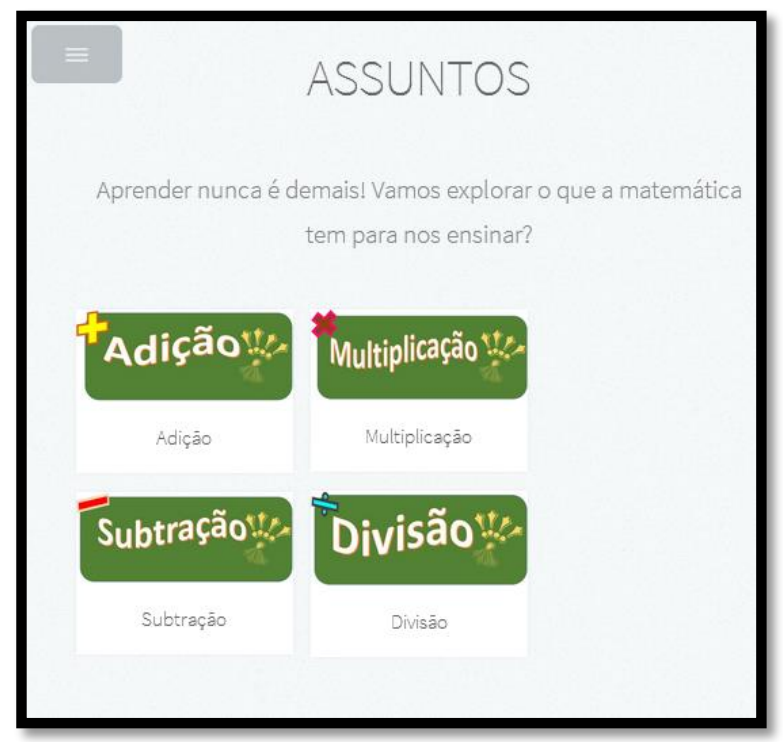

Figura 3. Tela de seleção da opção avançar ou Assuntos presente no Menu do sistema 


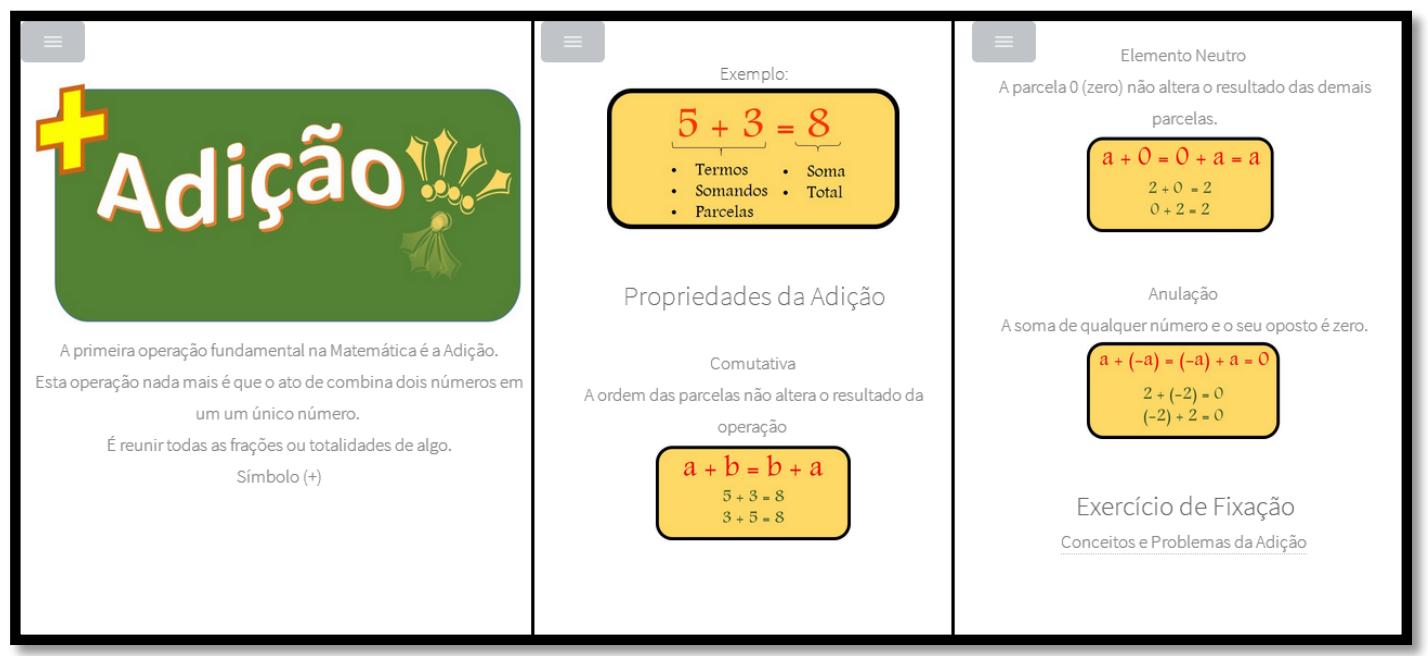

Figura 4. Tela com o conteúdo da opção selecionada em ASSUNTOS

Se a opção de Exercício de Fixação for selecionada, o sistema apresentará a tela com cinco questões objetivas para que o aluno responda, em seguida um pop-up aparecerá ao aluno, confirmando ou não se está pronto para receber sua pontuação. Se ele responder positivamente, instantaneamente outro pop-up aparecerá com a pontuação, como mostra a Figura 5 a seguir, sendo redirecionado à Tela inicial do sistema novamente.

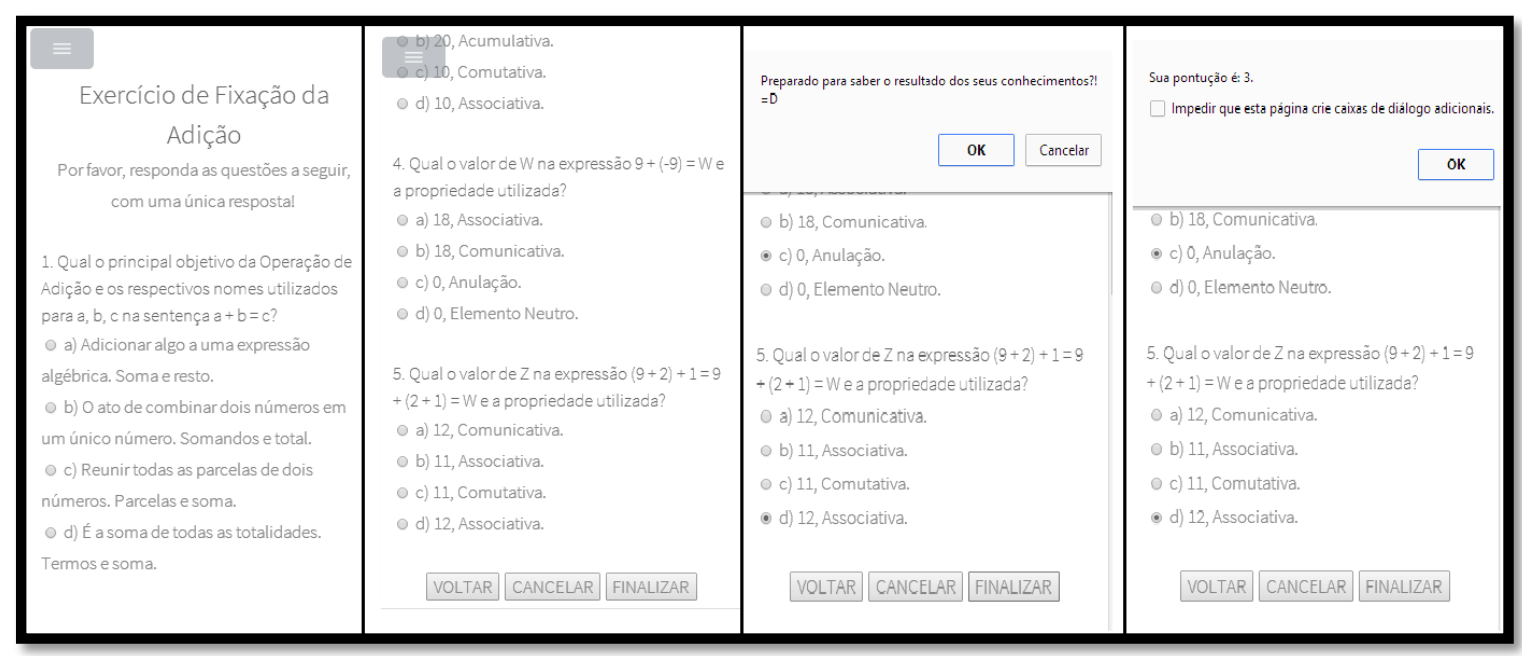

Figura 5. Tela de Exercício de Fixação com pop-ups de confirmação e pontuação

A última opção apresentada no Menu da aplicação é a de sites educacionais, uma iniciativa de apresentar outros links de sites que ajudam a aprendizagem de conteúdos diversos à matemática, ou seja, conteúdos diferenciado, aos quais os alunos não estão acostumados no seu cotidiano. Como exemplo, a Figura 6 a seguir mostra o link do site do Computação Desplugada ${ }^{5}$ e o redirecionamento da página web no sistema.

${ }^{5}$ http://icomp.ufam.edu.br/unplug/index.php?lang=pt-BR 


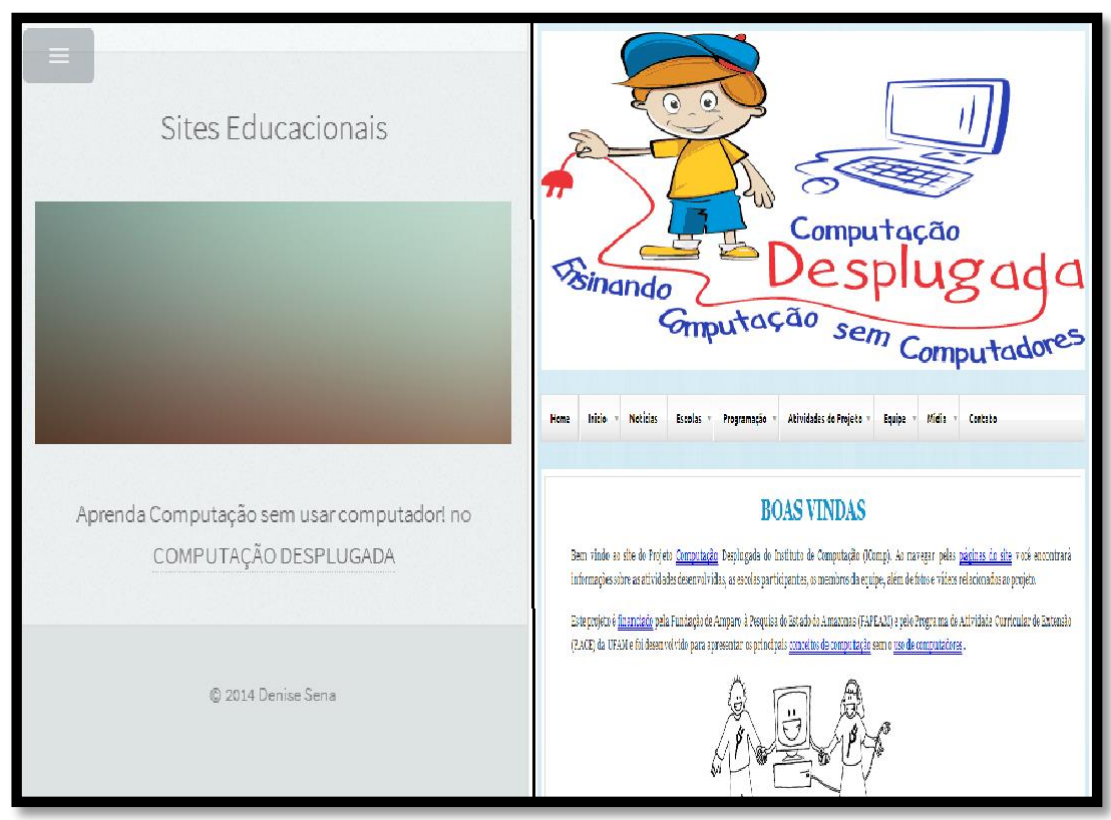

Figura 6. Tela da opção Sites Educacionais e o site redirecionado após a seleção

\section{Teste de Implantação e Resultados}

A realização do teste consistiu em tornar o Questions Today disponível por meio de uma versão beta, onde os alunos obtiveram o acesso a aplicação web via celular, capaz de prover conteúdos da disciplina de matemática e avaliá-los com os exercícios de fixação disponíveis.

Os alunos participantes da pesquisa, foram voluntários de uma escola da rede pública estadual localizada na cidade de Manaus, que cursavam a primeira série do ensino médio. Com um total de 41 alunos matriculados na turma da série escolhida para a realização da pesquisa, $83 \%$ (34 alunos) se prontificaram a participar, enquanto $17 \%$ ( 7 alunos) estavam realizando outras atividades na instituição ou não estavam presentes no momento.

Com a grande diversidade de dispositivos móveis existentes, não houve dificuldade com a falta de dispositivos para o acesso ao sistema, pois 83\% (34 alunos) dos alunos voluntários tinham celular para execução do sistema. No entanto, poucos tinham acesso à internet, e como meio de facilitar o acesso, foram instalados nos celulares o sistema de apoio a aprendizagem, para que todos obtivessem acesso e contribuir com a pesquisa.

O ambiente disponível para realizar a pesquisa foi uma sala de aula da instituição com um tempo de 45 minutos destinado para a realização do teste, e este foi dividido em 2 etapas:

1. Na primeira etapa, foram apresentados as etapas de realização da pesquisa, alguns conceitos básicos sobre aprendizagem móvel, o sistema de apoio a aprendizagem de matemática e a execução do sistema para que houvesse interação com o software apresentado e;

2. Na segunda etapa, cada aluno realizou a avaliação do sistema individualmente, que possuía 7 questões, para a análise dos resultados foi utilizado o método de 
frequência relativa em todas as 5 questões objetivas e obrigatórias, onde o aluno tinha que escolher as opções de: Concordo Totalmente (CT), Concordo Parcialmente (CP), Indiferente (IN) Discordo Parcialmente (DP) e Discordo Totalmente (DT), e 2 discursivas não obrigatórias, para análise futuras e contribuições de melhoria da aplicação. As principais questões são apresentadas na Tabela 1.

Tabela 1. Principais resultados da aplicação

\begin{tabular}{|l|c|c|c|c|c|}
\hline \multicolumn{1}{|c|}{ PERGUNTA } & CT & CP & IN & DP & DT \\
\hline Possui interesse pela Matemática? & $24 \%$ & $35 \%$ & $0 \%$ & $0 \%$ & $41 \%$ \\
\hline $\begin{array}{l}\text { As questões presentes no Questions Today, } \\
\text { ajudam no processo de aprendizagem? }\end{array}$ & $74 \%$ & $26 \%$ & $0 \%$ & $0 \%$ & $0 \%$ \\
\hline $\begin{array}{l}\text { Consegue identificar conteúdos dado pelo(a) seu } \\
\text { professor(a) em sala de aula? }\end{array}$ & $85 \%$ & $15 \%$ & $0 \%$ & $0 \%$ & $0 \%$ \\
\hline $\begin{array}{l}\text { É fácil utilizar o sistema Questions Today? } \\
\text { Usaria a aplicação Questions Today em seu } \\
\text { dispositivo móvel? }\end{array}$ & $100 \%$ & $0 \%$ & $0 \%$ & $0 \%$ & $0 \%$ \\
\hline
\end{tabular}

Em todas as etapas, a presença do aluno pesquisador foi primordial para esclarecer dúvidas e ajudar no processo de execução dos testes de implantação realizados com os alunos.

Com os resultados obtidos, $59 \%$ dos alunos responderam positivamente o interesse pela disciplina de matemática, sendo relevante os $41 \%$ que discordam totalmente relacionado as respostas concordo totalmente e parcialmente, pois a disciplina é a mais preocupante no ensino médio no meio dos alunos.

Com o intuito de descobrir se os alunos realizando uma auto avaliação, aprenderam ou não em pouco tempo, a questão "As questões presentes no Questions Today ajudam no processo de aprendizagem?", mostra o percentual máximo de $100 \%$ relacionada a resposta concordo totalmente e parcialmente, pois os resultados gerados pela aplicação da pontuação ao término dos exercícios, despertava um interesse nos alunos pela exploração de conhecimento que estavam disponíveis no sistema.

Desejando verificar a percepção dos alunos quanto aos seus conhecimentos obtidos, realizando a identificação de conteúdos aprendidos em sala de aula, obteve-se resposta positiva e maximal nas concordâncias, onde na questão "Consegue identificar conteúdos dado pelo(a) seu professor(a) em sala de aula?" apresentam $85 \%$ para concordo totalmente e $15 \%$ concordo parcialmente.

Para julgar se é fácil ou não utilizar o sistema, a questão "É fácil utilizar o sistema Questions Today?", mostra o quão favorável foi utilizá-lo, pois aponta que todos os participantes responderam de forma positiva sobre a usabilidade do sistema. 
Além disso, buscou-se ainda saber se os alunos utilizariam o aplicativo e seus dispositivos móveis e a aprovação foi total novamente.

Após a análise das questões objetivas, as informações das questões objetivas foram extraídas e analisadas, obtendo-se os resultados apresentados a seguir. Como existem diversos aplicativos para dispositivos móveis, foi feita a pergunta: "Você conhece outros Sistemas que podem ser utilizados no celular para ensinar matemática?". Apesar de não ser uma questão obrigatória, nem todos responderam e apenas 23,5\% (8 alunos) responderam a questão diferente de 'não', onde $62,5 \%$ (5 respostas) a calculadora foi mencionada, 37,5\% (3 respostas) mencionaram jogos e 76,5\% (26 alunos) disseram não conhecer outro aplicativo que ensine matemática.

Como melhorar o aplicativo, a questão "Que tipo de melhoria pode ser atribuído ao sistema Questions Today?" foi aplicada. Dos 34 alunos, 76,5\% (26 alunos) mencionaram que não será preciso melhoria e apenas 23,5\% (8 alunos) responderam a questão de forma diferente, onde $62,5 \%$ ( 5 respostas) pedem mais assuntos referentes a matemática, $12,5 \%$ ( 1 resposta) pede para mudar a cor, $12,5 \%$ (1 resposta) sugere a inclusão de uma calculadora para ajudar nos cálculos dos exercícios e 12,5\% (1 resposta) pede para acrescentar jogos.

Portanto, apesar de muitos alunos voluntários não se interessarem pela disciplina de matemática, houve a percepção da interatividade com um sistema móvel e o desejo pelo desenvolvimento da aprendizagem, a notória percepção de conhecimentos já assistidos, a facilidade de uso e o interesse pelo sistema em dispositivos de uso pessoal.

\section{Considerações Finais}

O artigo apresenta todo o processo de desenvolvimento do sistema m-learning Questions Today e uma análise da avaliação do teste de implantação do sistema em uma escola da rede pública, realizado com alunos do ensino médio.

Os resultados apresentados neste artigo levam-nos a acreditar que, o sistema Questions Today pode ser um importante meio de auxílio ao processo de ensino e aprendizagem de matemática. Esta pode ser mais uma forma de se utilizar os dispositivos móveis dos alunos do ensino médio das escolas públicas de maneira educativa e não apenas para entretenimento. Além desse modo de aprendizado de fazer com que os alunos resolvam problemas a partir de conteúdos apresentados, poderá servir como fator motivacional para os alunos aprenderem uma matéria com alto índices de rejeição, como é matemática.

Como trabalho futuro, pretende-se automatizar o processo de alimentação da base de conteúdos e geração de exercícios, assim como realizar cadastros de docentes e discentes para geração de histórico das atividades realizadas, para acompanhamento e monitoramento do processo de aprendizagem.

\section{Agradecimentos}

Part of the results presented in this paper were obtained through R\&D activities of "Large Scale Qualification Program on Mobile Technologies - PROMOBILE" project sponsored by Samsung Eletrônica da Amazônia Ltda. under the terms of Brazilian federal law No. 8.248/91.

À Fundação de Amparo à Pesquisa do Estado do Amazonas - FAPEAM, pelo 
apoio com o auxílio da bolsa para a Pós Graduação Stricto Senso - Mestrado.

\section{Referências}

Barcelos, G. T.; Batista, S. C. F. (2010) "Formação de Professores de Matemática: Uso Pedagógico das Tecnologias de Informação e Comunicação", In: X Encontro Nacional de Educação Matemática - Educação Matemática, Cultura e Diversidade, 7 - 9 jul. 2010. Salvador, BA.

Batista, S. C. F.; Behar, P. A.; Passerino, L. M. (2011) "M -learn Mat: Aplicação de um Modelo Pedagógico para Atividades de M-learning em Matemática", In: XXII Simpósio Brasileiro de Informática na Educação (SBIE)/XVII Workshop de Informática na Escola (WIE), 2011, 21 - 25 nov. 2011. Aracaju, SE.

Batista, S. C. F. (2011) "M -LearnMat: Modelo Pedagógico para Atividades de M learning em Matemática", Tese (doutorado em Informática na Educação). Porto Alegre, RS, Universidade Federal do Rio Grande do Sul - UFRGS, 2011.

Batista, S. C. F.; Behar, P. A., Passerino, L. M. (2010) "M -learning na Aprendizagem Matemática: investigando potencialidades e limitações”, In: Congreso Colombiano de Informática Educativa 20 Años (RIBIE-Col), 2010, Bogotá.

Batista, S. C. F.; Behar, P. A. (2009) "M-learning e Matemática: mapeando recursos e modalidades educacionais", RENOTE: Revista Novas Tecnologias na Educação, v. 7, n.2, Dez. 2009.

Batista, S. C. F.; Behar, P. A.; Passerino, L. M. (2010) "Recursos pedagógicos para dispositivos móveis - Uma análise com foco na Matemática", RENOTE: Revista Novas Tecnologias na Educação, v. 8 n. 3, Dez, 2010

Figueiredo, M. A.; Silva, O. G.; Costa, S. R. (2011) "Programas de Nivelamento de Matemática e Português: M -Learning com Videoaulas", In: Congresso Internacional de Educação a Distância - ABED (Associação Brasileira de Educação a Distância), Manaus, 2011.

Kitchenham, B. A.; Budgen, D.; Pearl Brereton, O. (2011) "Using mapping studies as the basis for further research - A participant-observer case study", Information and Software Technology, Volume 53, Edição 6, Jun, 2011.

Marçal, E.; Lima, L. de; Júnior, M.; Viana, W.; Andrade, R.; Ribeiro, J. W. (2010) "Da Elicitação de Requisitos ao Desenvolvimento de Aplicações de Mobile Learning em Matemática", In: Simpósio Brasileiro de Informática na Educação, João Pessoa Paraíba, 2010.

Neto, J. F. B.; Fonseca, F. de S. da. (2013) "Jogos educativos em dispositivos móveis como auxílio ao ensino da matemática", RENOTE: Revista Novas Tecnologias na Educação, v. 11, n 1, Jul, 2013.

Petersen, K.; Feldt, R.; Mujtaba, S.; Mattsson, M. (2008) Systematic mapping studies in software engineering. In Proceedings of the 12th international conference on Evaluation and Assessment in Software Engineering (EASE'08), Giuseppe Visaggio, Maria Teresa Baldassarre, Steve Linkman, and Mark Turner (Eds.). British Computer Society, Swinton, UK, UK, 68-77. 